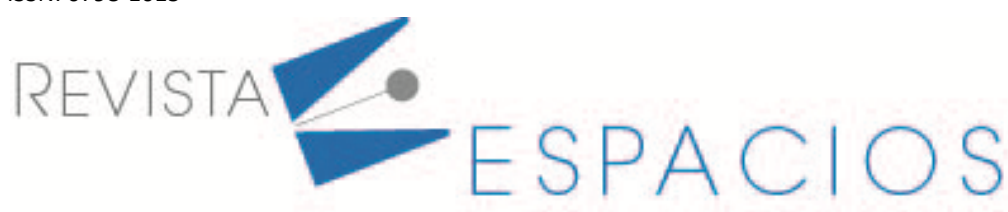

\title{
Ruteo con servicio tiempo dependiente y penalizaciones por espera y por pérdida de ventas
}

\section{Travelling salesman problem with time-dependent service and penaltys for waiting time and lost sales}

\author{
OSORIO, Cesar David ${ }^{1}$ \\ BRAVO-BASTIDAS, Juan José2 \\ OREJUELA, Juan Pablo 3
}

\begin{abstract}
Resumen
Se presenta un modelo para la optimización del ruteo en la recogida de ítems perecederos, optmizando el tiempo de inicio de la ruta, considerando tiempos de servicio y pérdidas de ventas tiempodependientes. Se consideran además los gastos asociados al mantenimiento del producto refrigerado, y se prueba el modelo en un caso real en un proceso de recogida, descargue en deposito y venta de leche en un municipio de Colombia, comprobando la utilidad del modelo con el uso de escenarios. Palabras clave: ruteo, proceso de recogida, tiempo de inicio óptimo, tiempo de servicio tiempo dependiente
\end{abstract}

\begin{abstract}
A model is presented for routing optimization in the collection of perishable items, optimizing the start time of the route, considering service times and time-dependent sales losses. The costs associated with the maintenance of the refrigerated product are also considered, and the model is tested in a real case in a process of collection, unloading and sale of milk in a region in Colombia, verifying the usefulness of the model with the use of scenarios.

key words: routing, pickup process, optimal start time, time dependent service time
\end{abstract}

\section{Introducción}

El problema del viajero de negocios es uno de los problemas de optimización combinatoria más reconocido y estudiado (Laporte, 2010), y consiste en encontrar el ciclo Hamiltoniano de peso mínimo en un grafo ponderado completo. Denominado como TSP (traveling salesman problem) este problema ha tenido amplia acogida en la investigación científica debido a su sencillez en el planteamiento pero a su complejidad en la solución, a tal punto que se cataloga como un problema NP-Hard (Gutin \& Punnen, 2003). Sin embargo, los problemas de ruteo que se enfrentan comúnmente en la práctica conllevan a complejizar también el planteamiento, como ocurre con las consideraciones de ventanas de tiempo (TSPTW) o los tiempos de servicio tiempo dependiente reconocido por sus siglas en ingléscomo TSP-TS y que será objeto de analisis en este artículo.

\footnotetext{
${ }^{1}$ Asistente de docencia. Escuela de ingeniería industrial. Universidad del Valle. cesar.osorio@correounivalle.edu.co

2 Profesor asociado. Escuela de ingeniería industrial. Universidad del Valle. juan.bravo@correounivalle.edu.co

${ }^{3}$ Profesor asociado. Escuela de ingeniería industrial. Universidad del Valle. juan.orejuela@correounivalle.edu.co
} 
Cada problema particular tiene un marco con elementos propios cuya relevancia es necesario considerar en cualquier esfuerzo de modelación, que si bien parte de la relativa sencillez del planteamiento del TSP básico como el que proponen Miller, Tucker \& Zemlin (1960), también requiere involucrar estructuras matemáticas que representen las particularidades del problema y que doten al modelo de representatividad. En el presente articulo se propone un modelo para la operación de recogidas refrigeradas, considerando la determinación del tiempo de inicio óptimo del tour, con una función piecewise (por partes) de perdida de ventas y de tiempos de descargue, tiempo dependientes que aún no han sido considerados en la literatura. El estudio del tiempo de inicio óptimo en problemas de recogidas refrigeradas, incluyendo el costo de espera de la unidad de refrigeración tampoco no se ha visto aún reflejado en la literatura científica junto con los otros elementos.

Tiempos de servicio tiempo dependientes. Los tiempos de servicio son un elemento que tradicionalmente no se ha abordado en la literatura respecto del TSP, puesto que el foco de atención se ha puesto en la minimización de la distancia total recorrida o de la duración total del tour, esta última considerada únicamente como el tiempo de los recorridos, o como la suma de dichos tiempos con los tiempos de servicio fijos en cada nodo., Esa naturaleza constante de los tiempos de servicio no requiere que ellos se involucren directamente en los procesos de optimización, sino que basta con que al tiempo total de recorrido mínimo obtenido, se le sume esa cantidad constante asociada con dichos tiempos. Fue solo hasta el 2016 que Taş, Gendreau, Jabali \& Laporte hacen la primera propuesta en la literatura en la que se plantea genéricamente el TSP con tiempos de servicio tiempo dependientes (TSP-TS) desarrollando un modelo de programación entera mixta para resolverlo, en el cual sí es necesario involucrar los tiempos de servicio en cada nodo como una función del tiempo de llegada al nodo, ya que el TSP-TS busca minimizar el tiempo total definido como la suma del tiempo de los viajes y los tiempos de servicio.

Según Cacchiani, Contreras-Bolton, \& Toth (2020) en muchas situaciones prácticas del TSP la consideración del tiempo de servicio tiempo dependiente es relevante, ya que factores como el personal disponible para el cargue/descargue de vehículos o la dificultad de acceso a algunos nodos en determinadas horas del día, hacen que los tiempos de servicios difieran de ciertas horas a otras, invalidando el supuesto de tiempos constantes y aportando complejidad al problema. Según Taş et al (2016), la consideración de la dependencia temporal conlleva a la definición de una función de tiempos de servicio que generalmente es no lineal y que debe involucrarse en el modelo al cual también se le adiciona un conjunto de variables $b_{i}$ definidas como el tiempo de llegada al nodo i, así como el conjunto de restricciones que rigen el comportamiento de estas, teniendo como resultado un modelo más robusto y no lineal que evidencia la complejidad del tipo NP-Hard del problema (Cacchiani et al., 2020).

Problema del tiempo de inicio óptimo (Optimal Start Time Problem). Los problemas de ruteo donde se consideran parámetros dependientes del tiempo, requieren de un seguimiento estricto de la evolución de este desde el momento de inicio del tour, ya que dicha evolución es la que va determinando los valores de los parámetros. Por ejemplo, en el caso del ruteo tiempo dependiente el parámetro de los tiempos de viaje entre nodos depende del momento en el que inicia el viaje, lo cual suele representarse a través de una función $c_{i j}\left(t_{i}\right)$ indicando que el tiempo de viaje del nodo i al nodo j depende del tiempo en el que se sale de un nodo $\mathrm{i}\left(t_{i}\right)$ (Malandraki \& Daskin, 1992). Se asume que dicha función ya está definida y por consiguiente, cada tiempo de partida $t_{i}$, para todo nodo distinto al depósito, se determina según sea el tiempo de inicio del tour (tiempo de partida del depósito), haciendo esto que el problema del seguimiento temporal se reduzca a conocer el tiempo de inicio del tour.

En este orden de ideas, los parámetros tiempo dependientes en los problemas de ruteo inducen la necesidad de considerar variables para el seguimiento temporal y de determinar el tiempo de inicio óptimo del tour. Hashimoto, Yagiura, \& Ibaraki (2008) formalizan ese problema de encontrar el tiempo de inicio dada una ruta, 
de manera que se minimicen los costos, y lo denominan como "Optimal Start Time Problem". En este artículo en que la dependencia de tiempo es respecto al tiempo de servicio, se tiene precisamente la necesidad de contar con un seguimiento temporal y de determinar el tiempo de inicio óptimo del tour.

Penalización por pérdida de ventas. Según Ballou (2004) la pérdida de ventas es una variable importante a considerar en la estructura de costos de la operación logística del suministro de productos. Para Mukhopadhyay \& Goswami (2016) la pérdida de ventas conlleva a muchas empresas a grandes pérdidas no solamente monetarias, sino intangibles como la reputación, realizandose grandes esfuerzos operativos y de capital para mitigar los stockouts o eventos de falta de inventario. Es por esta importancia del costo de perdida de ventas y el importante esfuerzo requerido para su reducción, que Ballou (2004) menciona la necesidad de determinar el equilibrio óptimo entre ese costo y otros costos de logística como de inventario y transporte, puesto que entre estos ocurren conflictos en donde una reducción en las ventas perdidas requiere un aumento en los costos de inventario y/o de transporte, siendo en muchos casos muy costoso tener un nivel de servicio del $100 \%$ o muy cercano a éste. Sobre la búsqueda de ese equilibrio óptimo respecto a problemas de ruteo en cadenas de suministro, la literatura muestra modelos que involucran una penalización por pérdida de ventas (Giesen, Mahmassani, \& Jaillet, 2005, 2010; Moosavi \& Nikfarjam, 2019).

\section{Planteamiento del problema}

El problema abordado en este paper consiste en el ruteo para la recogida de productos refrigerados sobre un conjunto de nodos dispersos geográficamente. Dicho ruteo se aborda desde el TSP, es decir, se considera un único vehículo que parte de un depósito hacia ciertos nodos, visitándolos a todos una única vez para realizar las recogidas y regresando de nuevo al depósito donde se requieren comercializar los productos recolectados. Este tipo de escenarios surgen, por ejemplo, en negociaciones en las que el comprador se encarga de recoger los productos en donde el proveedor los almacena, que en términos de las reglas de Inconterms, que rigen las operaciones de compraventa tanto nacionales como internacionales, se conoce como "En Fábrica" o en inglés "Ex Works" (EXW).

En el contexto de los problemas de recogidas, algunos depósitos tienen horarios de operación donde se realiza la venta del producto previamente recogido, lo cual define una ventana de tiempo relevante. Esta empieza con la hora de inicio de descargue de vehículos y termina con la hora en la que se cierra el establecimiento. Cuando los vehículos recolectores llegan de maneraanticipada, es decir, llegan antes de la hora de inicio de descargue (inicio de la ventana), se incurre en una espera que implica el funcionamiento de la unidad de refrigeración del vehículo, generando costos por consumo de combustible que impactan la eficiencia de la operación y exigiendo por ello una mayor sincronía en el proceso.

En la mencionada ventana de tiempo del depósito, en la práctica es muy común observar que en las vecindades de la hora de inicio de descargue suele ocurrir una alta congestión de vehículos que esperan ser atendidos para comercializar sus productos, y por esa alta congestión el tiempo de servicio a los vehículos en esa franja horaria es mayor que en otros momentos, situación que sugiere considerar la dependencia del tiempo de servicio en el depósito (descargue de productos) en función del momento de arribo a él.

Adicionalmente a la hora de inicio de descargue, en este tipo de depósitos de comercialización se define una hora de inicio de ventas o de comercialización, que es el momento de apertura a los clientes, y ello resalta la ocurrencia de ciertas perdidas de ventas en función de la tardanza del descargue del vehículo frente a esa hora de inicio de comercialización en el depósito. Además, es claro que el descargue de un vehículo después del momento de cierre del deposito implicaría una pérdida de ventas máxima, ya que en esas horas el depósito no opera, es decir, no hay comercio. Con esa misma idea, la pérdida de ventas es nula cuando el producto se descarga en el momento de inicio de la comercialización o antes, puesto que en esas horas el producto logra 
estar disponible desde el momento en que la demanda inicia. Pero si el producto no llega en dicho inicio o antes, a medida que transcurre el tiempo se va dejando de atender demanda potencial, incurriendo en perdida de ventas.

En tal sentido, el problema abordado en este trabajo consiste en un TSP de recogidas de productos refrigerados para su comercialización una vez se llegue al depósito, considerando los tres elementos discutidos en la sección anterior: tiempos de espera, tiempos de servicio (tiempo de descargue) tiempo dependientes y penalización por pérdida de ventas, los cuales serán componentes del costo total que también incluye el costo por consumo de combustible en el desplazamiento entre los nodos proveedores. El objetivo será entonces la minimización de ese costo total sobre el cual, el funcionamiento de la unidad de frio tiene un impacto significativo, toda vez que es un factor de costo relevante cuando los arribos se dan antes de la hora de inicio de descargues, y durante el tiempo de servicio. Por otro lado, se asume que se incurre en un costo por perdida de ventas solo cuando la finalización del descargue del vehículo ocurre después de la hora de inicio de la comercialización de productos.

La Figura 1 ilustra el funcionamiento de los costos asociados a la espera, los tiempos de servicio y la pérdida de ventas, en donde la línea verde representa la hora en la que se inician los descargues de los vehículos en el deposito, que se denota como "hid", la línea azul representa la hora en la que se inicia la comercialización de los productos, denotada como "hic", y la ubicación de los camiones en dicha figura simboliza el momento de arribo al depósito ("TL") en cada uno de los cinco escenario presentados.

En el caso A el vehículo arriba antes de que se inicien los descargues de vehículos (línea verde), y en función de ese momento de llegada se le asocia un tiempo de servicio " $\mathrm{ts}$ " el cual empieza a transcurrir después de la espera "TE", es decir, una vez se llegue a la "hid"; en términos de costos se tiene que sobre el tiempo "TE" se incurre en refrigeración, así mismo en el tiempo de descargue "ts", y el costo por pérdida de ventas ocurrirá solamente si la finalización del tiempo de servicio o de descargue ("TD") ocurre después de la "hic" (línea azul), que para el caso A no se tiene esa situación, como se puede observar en la Figura 1.

El caso B es un caso similar al A, en el que el arribo del vehículo se da antes de la "hid" pero además de los costos de refrigeración asociados al "TE" $y$ el "ts", se incurre en un costo por pérdida de ventas "pv" debido a que el "TD" es posterior a la "hic". El caso C y D son situaciones en que el arribo ("TL") ocurre después de la "hid" pero antes de la "hic", y muestran las opciones de que el "TD" ocurra antes de la "hic" (caso C) o después (caso D), teniendo en el primero solamente costos de refrigeración sobre el "ts" y en el segundo, además de los costos de refrigeración, los costos por pérdida de ventas. Por último, la Figura 1 muestra el caso E en el que el arribo se da después de la línea azul, por lo que tiene costos asociados al tiempo de servicio asignado en función de su hora de llegada y a la pérdida de ventas. 
Figura 1

Costos del problema

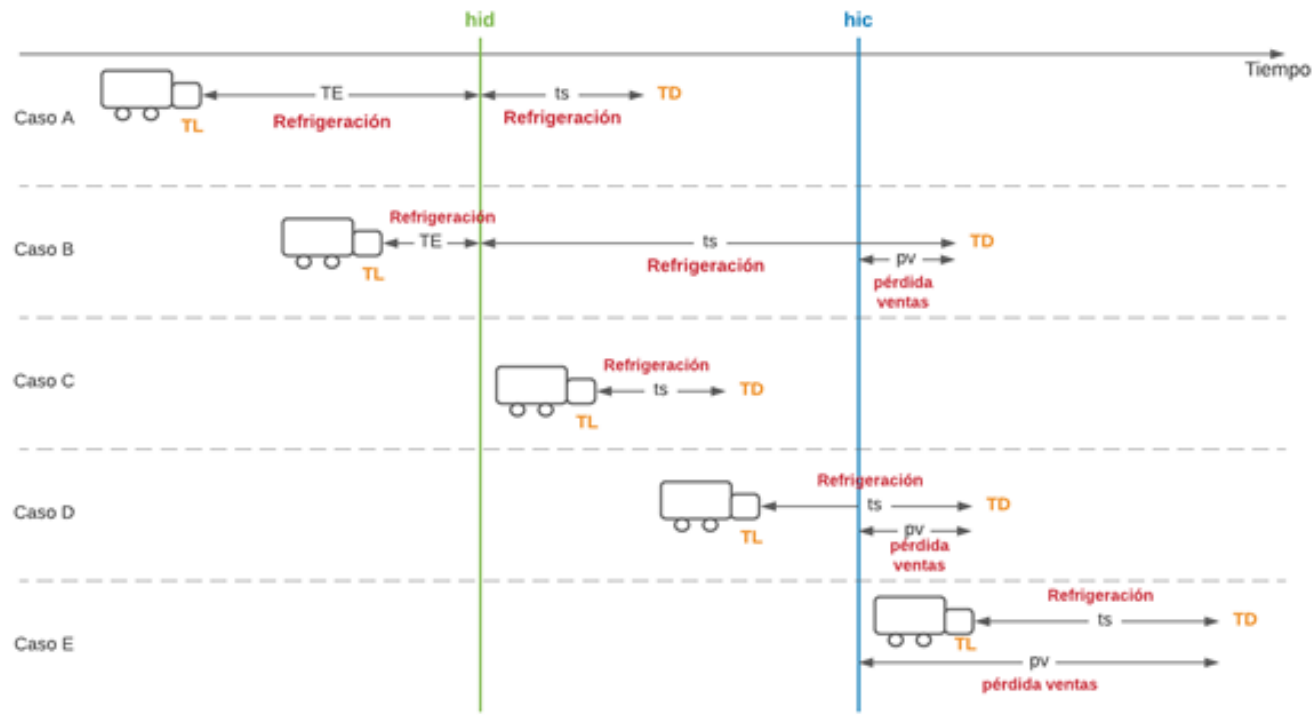

La descripción del problema en la Figura 1 contiene una aproximación a la naturaleza de los elementos de costo que componen la función de desempeño planteada en este trabajo, específicamente sobre los componentes de costo por espera, penalización por tiempo de servicio y penalización por pérdida de ventas. Respecto a la espera, el costo es función del tiempo de llegada al depósito ( $T L)$, puesto que si el arribo ocurre antes de la hora de inicio de descargue (hid), entonces el tiempo de espera se define como hid-TL, siendo el costo directamente proporcional a ese tiempo de espera, ya que es la multiplicación de este por el consumo de la unidad de frio en términos monetarios, de manera que, matemáticamente el costo por espera es una función lineal dependiente de TL con pendiente negativa que llega a cero en el tiempo igual a "hid" tal como se representa en la recta verde de la Figura 2.

Por otro lado, la descripción del problema sugiere que la penalización por tiempo de servicio también es una función del tiempo de llegada (TL), sin embargo, el comportamiento de ésta no es lineal como en el caso anterior, sino que corresponde a una función por partes en donde a unos determinados rangos del tiempo de llegada se le asocia un tiempo de servicio que se monetiza multiplicándose por el consumo de la unidad de frio, resultando la función representada en rojo en la Figura 2.

Figura 2

Costos en función del tiempo de llegada.

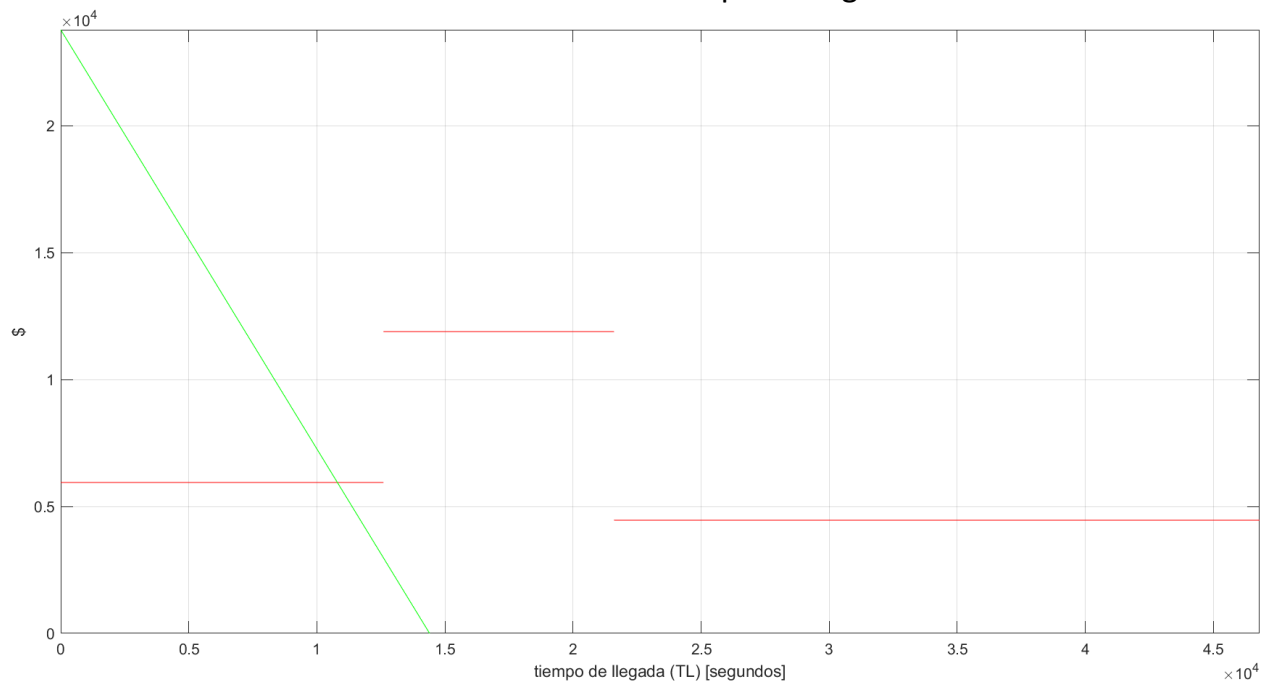


La interacción de las funciones mostradas en la Figura 2, así como la no linealidad que se evidencia en ella, son una complejidad que se acentúa aún más con la consideración adicional de la penalización por perdida de ventas antes mencionada, y cuya naturaleza tampoco es lineal sino que consiste en una función por partes dependiente del tiempo de finalización del descargue (TD). Esto se debe a que únicamente en el instante en que se finaliza el descargue es cuando se tiene disponibilidad de los productos para su comercialización y, como se muestra en la Figura 1, si el TD ocurre después de la hora de inicio de comercialización (hic), entonces la tardanza se define como TD-hic y en función de esta tardanza es que se dan las ventas perdidas en una relación directa. En la Figura 3 se observa que la función tiene como dominio el intervalo entre la hora de inicio de la comercialización y la hora de cierre del depósito y que a medida que el tiempo de descargue crece, también lo hace la penalización en segmentos de recta que definen a la función por partes, tal que la pendiente de los segmentos crece a medida que los intervalos corresponden a mayores tiempos.

Figura 3

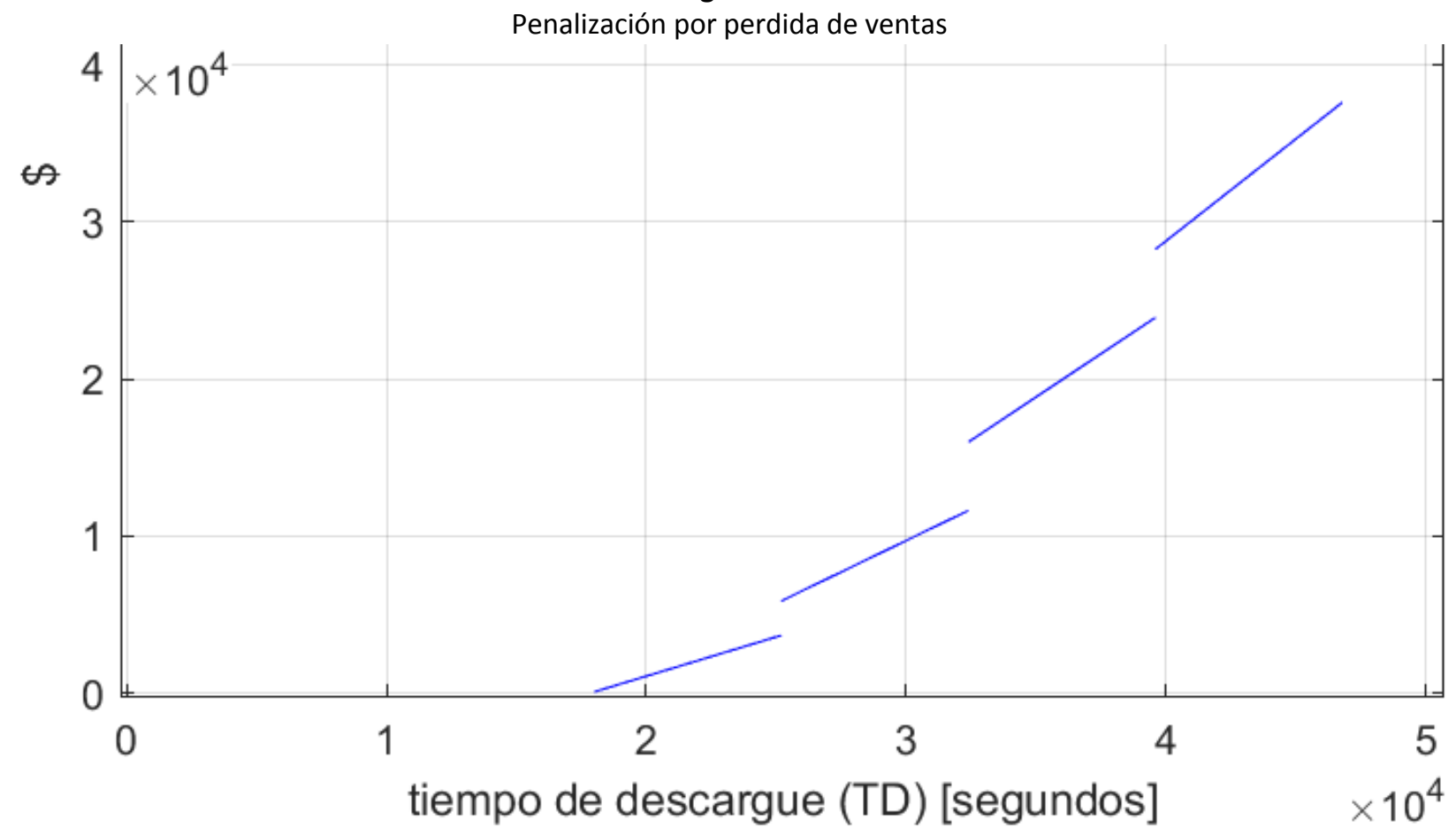

\section{Metodología}

En esta sección se propone un modelo matemático como representación del problema descrito en la sección anterior que consiste en determinar tanto la secuencia de nodos de recolección a visitar, como el momento de inicio del tour, tal que los costos totales incurridos sean mínimos.

El modelo propuesto está diseñado con elementos que aún no han sido combinados en la literatura como la función piecewise de perdida de ventas y de tiempos de descargue, tiempo dependientes. El estudio del tiempo de inicio óptimo en problemas de recogidas refrigeradas, incluyendo el costo de espera de la unidad de refrigeración no se ha visto aún reflejado en la literatura.

El manejo de sub-tours se basa en la propuesta de Miller, Tucker \& Zemlin(1960) y las estructuras para representar los intervalos de las funciones por partes se inspiran en el trabajo de Orejuela \& Hernández (2019). Se trata pues de un modelo de programación lineal entera mixta, presentado a continuación, donde la estructura 
de la función objetivo se compone de las funciones representadas en la Figura 2 y Figura 3, además del consumo de combustible asociado al recorrido, que junto con las restricciones inherentes al problema, son el aporte fundamental del presente artículo. Respecto a la relevancia de la evolución temporal en el problema, se definen variables de tiempo como $T_{0}, T L$ y $T D$, y particularmente con la variable del tiempo de inicio $\left(T_{0}\right)$ se resuelve el OSTP (Optimal Start Time Problem, por su nombre en inglés) propuesto por Hashimoto, Yagiura, \& Ibaraki (2008) para problemas tiempo dependientes.

\section{Conjuntos:}

$\operatorname{Nodos}[N O]=\{0,1,2, \ldots, n\}$, indices $i, j$

Intervalos de tiempos de servicio $[K]=\{1,2, \ldots, m\}$, indice $k$

Intervalos de perdida de ventas $[H]=\{1,2, \ldots, l\}$, indice $h$

\section{Parámetros:}

$t_{i j}=$ tiempo de viaje del nodo $i$ al nodo $j[\mathrm{seg}]$

liits $_{k}=$ limite inferior del intervalo de tiempos de servicio $k[\mathrm{seg}]$

$l_{\text {sits }}=$ limite superior del intervalo de tiempo de servicio $k$ [seg]

liipv $v_{h}=$ limite inferior del intervalo de pérdida de ventas $h$ [seg]

$l \operatorname{sip} v_{h}=$ limite superior del intervalo de pérdida de ventas $h$ [seg]

cguf = consumo de gasolina de la unidad de frio $\left[\frac{\text { litros }}{\mathrm{seg}}\right]$

cgr $=$ consumo de gasolina por tiempo de recorrido $\left[\frac{\text { litros }}{\mathrm{seg}}\right]$

$c d g=$ costo de gasolina $\left[\frac{\$}{\text { litro }}\right]$

$p p v_{h}=$ penalización fija por pérdida de ventas asociada al intervalo $h$

pend $_{h}=$ pendiente de la función de pérdida de ventas en el intervalo $h$

tst $d_{k}=$ tiempo de servicio en el depósito en el intervalo $k[\mathrm{seg}]$

hid $=$ hora de inicio de descargues $[\mathrm{seg}]$

Variables:

$X_{i j}=\left\{\begin{array}{l}1, \text { si se realiza el recorrido del nodo } i \text { al nodo } j \\ 0, \text { si no }\end{array}\right.$

$U_{i}=$ variable para subtours

$T_{0}=$ tiempo de partida del depósito (inicio del tour)

$T L=$ tiempo de llegada del depósito

$T D=$ tiempo en el que el vehículo es descargado en el depósito

$T A I D=$ tardanza respecto a hora de incio de descargue (hid)

ANID = anticipo o tiempo de espera respecto a hora de inicio de descargue (hid)

$T A I C=$ tardanza respecto al inicio de la cormercialización en el depósito

ANIC $=$ anticipo respecto al inicio de la comercialización en el depósito

$\mathrm{TAICH}_{h}=$ variable auxiliar para asociar la dimensión de TAIC a un intervalo $h$ 


$$
\begin{aligned}
& B_{h}=\left\{\begin{array}{l}
1, \text { si TAIC } \in \text { intervalo } h \\
0, \text { si no }
\end{array}\right. \\
& Y_{k}=\left\{\begin{array}{l}
1, \text { si } T L \in \text { intervalo } k \\
0, \text { si no }
\end{array}\right. \\
& \text { Función objetivo: } \\
& \operatorname{Min} f=\operatorname{cgr} * \operatorname{cdg}\left[\sum_{i \in \mathbb{N} O} \sum_{j \in N O, i \neq j} X_{i j} * t_{i j}\right]+\operatorname{cguf} * \operatorname{cdg}\left[\sum_{i \in N O} \sum_{j \in \mathbb{N O}, i \neq j} X_{i j} * t_{i j}+A N I D+\sum_{k \in K} Y_{k} * t s t d_{k}\right]+\sum_{h \in H}\left(T A I C H_{h} * \operatorname{pend}_{h}+B_{h} * p p v_{h}\right) \\
& \text { Restricciones: } \\
& \sum_{i \in N O, i \neq j} X_{i j}=1 ; \quad \forall j \in N O \\
& \sum_{j \in N O, i \neq j} X_{i j}=1 ; \quad \forall i \in N O \\
& U_{i}-U_{j}+n X_{i j} \leq n-1 ; \quad \forall i, j \in N O-\{0\}, i \neq j \\
& T L=T_{0}+\sum_{i \in N O} \sum_{j \in N O, i \neq j} X_{i j} t_{i j} \\
& T L-\text { hid }=T A I D-A N I D \\
& T D=h i d+\sum_{k \in K} Y_{k} * t s t d_{k}+T A I D \\
& T D-l i i p v_{1}=T A I C-A N I C \\
& T A I C=\sum_{h \in H} T A I C H_{h} \\
& B_{h}\left(\operatorname{lsipv}_{h}-\operatorname{liipv}_{1}\right) \geq \text { TAICH }_{h} ; \quad \forall h \in H \quad \text { (10) } \\
& \sum_{h \in H} B_{h} \leq 1 \\
& \sum_{k \in K} Y_{k} * \text { liits }_{k} \leq T L \\
& \sum_{k \in K} Y_{k} * l s i t s_{k} \geq T L
\end{aligned}
$$

El primer término de la función objetivo corresponde a los costos por consumo de gasolina durante el tiempo del recorrido de los vehículos, el segundo término se asocia al costo por consumo de gasolina de la unidad de refrigerado, la cual trabaja durante el recorrido, además del tiempo de espera por el anticipo respecto al inicio del descargue representado por la variable ANID (en la Figura 1 se muestra como TE), y durante el tiempo de descargue del nodo que depende del intervalo $\mathrm{h}$ en que el vehículo arribó (ver Figura 1); el tercer término corresponde a la penalización por pérdida de ventas la cual está en función de la tardanza respecto a la hora de inicio de la comercialización representada por la variable TAICH que informa sobre la magnitud de la tardanza, así como sobre el intervalo en el que se da, ya que la penalización se compone de un elemento fijo dependiente del intervalo, y un componente variable dependiente de la magnitud.

Respecto a la restricciones se tiene que la (1) y (2) indican que a cada nodo se debe llegar y se debe partir una única vez, conforme a la naturalezas del TSP; (3) corresponde a la restricción del rompimiento de sub-tours 
propuesta por Miller et al. (1960); en (4) se define que el tiempo de llegada al depósito y corresponde a la hora de inicio del tour más la duración del mismo; (5) determina la tardanza o el anticipo del tiempo de llegada respecto a la hora de inicio de descargues; la expresión (6) define el momento en que el vehículo termina de descargarse (TD) en función de la hora de inicio del descargue, el tiempo de servicio tiempo dependiente y la tardanza respecto a dicha hora de inicio del descargue, tal como se discute en la Figura 1; (7) calcula la tardanza o el anticipo respecto al inicio de la comercialización; en (8), (9) y (10) se asocian con la magnitud de la tardanza frente a la comercialización (TAIC), a un intervalo h; (11) garantiza que a lo sumo exista un intervalo h asociado a TAIC; en (12) y (13) se define el intervalo k asociado al tiempo de llegada TL; (14) garantiza que se active a lo sumo un intervalo k; y la restricción (15) garantiza que el tiempo de descargue siempre sea menor a la hora en la que en el depósito se cierra.

\section{Resultados de aplicación}

En esta sección se describe detalladamente el caso de estudio y los resultados de la metodología de solución propuesta. Posteriormente, como análisis de resultados, se plantea un análisis de sensibilidad en el marco de tres escenarios para identificar el impacto sobre la función objetivo de situaciones como: cambios tecnológicos en el vehículo en consideración (escenario 1), variaciones en la disponibilidad de mano de obra para descargue (escenario 2) y cambios en las percepciones de los clientes frente a bajos niveles de servicio (escenario 3 ). Finalmente, se realiza un análisis del impacto del momento de inicio en los costos totales del problema, con el objetivo de mostrar la importancia de la consideración del momento de inicio del tour, en este tipo de problemas.

\subsection{Caso de estudio}

En el contexto colombiano, un ejemplo de ello es el caso de la comercialización de leche cruda (sin procesamiento) producida por los campesinos de Cumbal, municipio del departamento de Nariño, los cuales venden una parte de la producción a empresas de procesamiento de leche, mientras que la producción restante puede ser comercializada en la galería municipal de Cumbal, para lo cual se requiere una recogida refrigerada del producto que garantice la cadena de frio requerida y que lleve el producto hasta el punto de venta en la galería. Este reto logístico es compatible con el modelo antes formulado aplicable a perecederos y en cuya aplicación práctica se tendría que el depósito corresponde a la galería donde se comercializa la leche, cuyos horarios de atención se representan con la ventana de tiempo discutida anteriormente. En este marco cobra sentido y relevancia la consideración de los costos asociados a la espera refrigerada, los tiempos de servicio tiempo dependientes y los costos por pérdida de ventas. Por consiguiente, dicha situación del municipio de Cumbal se propone como caso de estudio real para la aplicación de la metodología propuesta a la solución del problema.

En lo que resta de esta sección se realiza una descripción del caso de estudio basada en la investigación realizada por Riascos \& Taimal (2019).

El municipio de Cumbal se encuentra ubicado en el sur occidente del departamento de Nariño, cuenta con una economía fundamentalmente agropecuaria, y su ubicación geográfica favorece principalmente la producción de leche. Esta actividad lechera se lleva a cabo fundamentalmente por productores organizados en asociaciones, las cuales venden aproximadamente el $75 \%$ de su producción a empresas procesadoras como Alpina, Colácteos y Alquería, y el $25 \%$ restante de la leche cruda requiere venderse en otros mercados (queseras o consumidor final) antes de que se cumpla el ciclo de vida del producto.

Las asociaciones adquieren enfriadoras con el objetivo de poder almacenar su producción diaria en las condiciones de frio necesarias. Según Riascos \& Taimal (2019) hasta esa fecha existían 33 enfriadoras de leche; cada enfriadora es propiedad de los asociados pero recibe producto tanto de estos como de proveedores 
(productores no asociados). Un operario es quien realiza la recepción de la leche y la dispone en el tanque frio para mantenerla en condiciones favorables. El número aproximado de socios por cada enfriadora son 43, generalmente con 10 proveedores. El proceso comienza en las fincas de los productores (socios y proveedores) los cuales realizan el ordeño y transportan el producto hasta la enfriadora de su asociación, y una vez el producto llega a la enfriadora se almacena en tanques de frio a una temperatura de $4^{\circ} \mathrm{C}$.

Una de las principales problemáticas que enfrentan las enfriadoras es la brecha existente entre la oferta y la demanda, puesto que, como se mencionó anteriormente, aproximadamente el $25 \%$ de la leche almacenada en las enfriadoras no es comprada por los grandes clientes (plantas procesadoras), haciendo necesaria la búsqueda de otras alternativas para su venta, entre las cuales está la comercialización de la leche cruda en la galería municipal de Cumbal (Figura 4), lo cual requiere una gestión de recogidas eficiente que permita obtener un beneficio económico en esa búsqueda de expansión de mercados..

Figura 4

Galería municipio de Cumbal

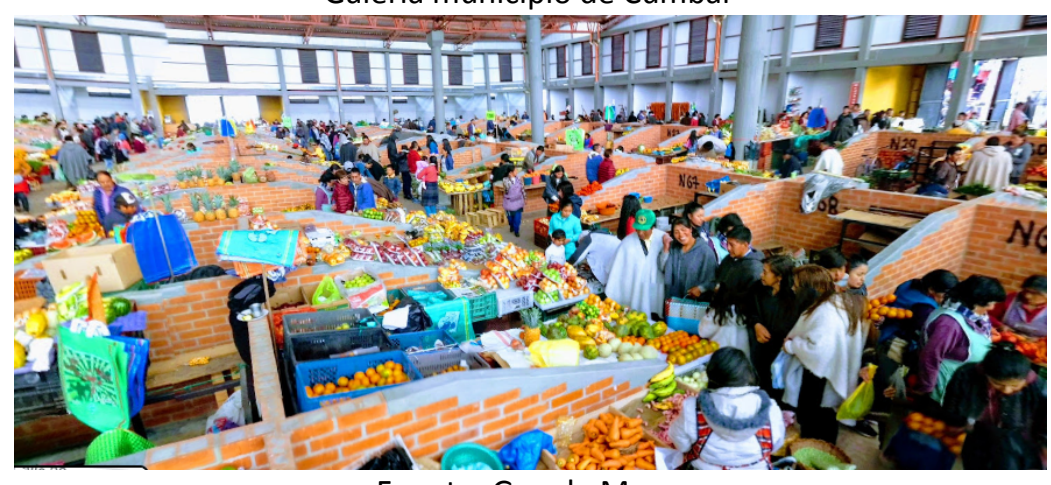

Fuente: Google Maps

Para el proceso de recogidas abordado en este caso de estudio, se van a considerar las coordenadas geográficas de 26 enfriadoras que Riascos \& Taimal (2019) recolectaron en su investigación. La distribución geográfica en la zona rural del municipio de Cumbal se puede observar en la Figura 5 donde los puntos verdes corresponden a las enfriadoras y el punto rojo a la galería.

Para garantizar una adecuada calidad del producto y aumentar su vida útil, se requiere que tanto la recogida en las enfriadoras como el almacenamiento en la galería se haga de una manera refrigerada para conservar la cadena de frio que evita la rápida reproducción de microorganismos. Así que el proceso de recolección de la leche cruda se debe realizar en un vehículo refrigerado el cuál visita a cada una de las enfriadoras recogiendo el producto para posteriormente llevarlo a la galería para su comercialización. En este escenario, el depósito del tour es la galería y a ésta se le asocia una ventana de tiempo que empieza con la hora de inicio de los descargues de los vehículos (4:00 h o $14400 \mathrm{seg}$ ) y finaliza con la hora en la que se cierra el establecimiento (13:00 h o 46800 seg).

A partir de dicha ventana de tiempo surgen también otras particularidades del caso que son importantes en el ejercicio del ruteo en la recogida y que se plantean a continuación.

\section{Costos de la espera refrigerada}

Al tener una recogida refrigerada y considerando la existencia de una hora específica en la que se inician los descargues de los vehículos en la galería, toma especial importancia la variable de adelanto o anticipo, definida como la diferencia entre la hora de inicio de descargue y la hora de llegada del camión, que se representa con la variable ANID del modelo matemático propuesto en la metodología. La importancia de este tiempo radica en que si el camión llega antes de la hora de inicio de descargue (situación en la que la variable ANID toma valores 
según la restricción 5 del modelo), debe esperar a que llegue el momento de inicio de descargue y como la espera debe ser refrigerada, entonces existe un costo asociado al trabajo de la unidad de refrigeración del vehículo durante ese tiempo.

Cabe resaltar que el costo del uso de la unidad de refrigerado no ocurre únicamente en la mencionada espera, sino también en el recorrido mismo, y los parámetros necesarios para su cálculo son el consumo de gasolina de la refrigeración por unidad de tiempo, que es de $6.944 \times 10^{-4} \mathrm{It} / \mathrm{seg}$ y el costo de la gasolina que se asume de $2377.55 \$ /$ lt, cuyos valores se definen según (AEOC, 2017).

\section{Tiempo de servicio tiempo dependiente}

Adicionalmente, al tratarse de una galería en la que confluyen vehículos con productos para ser comercializados, ocurre que en las vecindades de la hora de inicio de los descargues, hay una alta congestión de vehículos para ser descargados, por lo que el tiempo de descargue de un vehículo que recoge la leche depende de la hora en la que arribe al depósito. El modelo propuesta enfrenta esta situación, con el vehículo considerado, y propone una función de tiempo de servicio como la mostrada en la curva roja de la Figura 2, en donde en cada intervalo se define una constante, es decir, hay un único tiempo de servicio asociado a cada intervalo, cuyos valores se detallan en la Tabla 1, sobre la cual se evidencia que el tiempo de servicio es mayor cuando la llegada del vehículo ocurre entre las 3:30 h y las 6:00 h, puesto que en esa franja se da la hora de inicio de descargue (4:00 ho 14400 seg), y alrededor de dicha hora es donde se espera mayor congestión.

Tabla 1

Función tiempo de servicio

\begin{tabular}{|c|c|c|}
\hline $\begin{array}{c}\text { Límite inferior } \\
\text { del intevalo [seg] }\end{array}$ & $\begin{array}{c}\text { Límite superior del } \\
\text { intervalo [seg] }\end{array}$ & $\begin{array}{c}\text { Tiempo de servicio } \\
\text { [seg] }\end{array}$ \\
\hline $0(0: 00 \mathrm{~h})$ & $12600(3: 30 \mathrm{~h})$ & 3600 \\
\hline $12600(3: 30 \mathrm{~h})$ & $21600(6: 00 \mathrm{~h})$ & 7200 \\
\hline $21600(6: 00 \mathrm{~h})$ & $46800(13: 00 \mathrm{~h})$ & 2700 \\
\hline
\end{tabular}

\section{Penalización por pérdida de ventas}

Por otro lado, al tratarse de una comercialización del producto recogido que inicia una vez el camión es descargado, a medida que ese momento de descargue (variable TD del modelo matemático) ocurra después de la hora de inicio de la comercialización en la galería (5:00 h o $18000 \mathrm{seg})$, entra a jugar un costo asociado a la pérdida de ventas por falta de disponibilidad del producto, que se expresa en el modelo como una función por partes, pero con la diferencia de que los segmentos asociados a cada intervalo no corresponden a una constante sino a una función lineal con pendiente positiva, tal como se observa en la Figura 3, ya que entre mayor sea la tardanza del descargue frente al inicio de la comercialización, mayor es la pérdida de ventas. Los datos tanto de la pendiente como de la constante del segmento de recta para cada intervalo se presentan en la Tabla 2. 
Tabla 2

Función pérdida ventas

\begin{tabular}{|c|c|c|c|}
\hline $\begin{array}{c}\text { Límite inferior del } \\
\text { intervalo [seg] }\end{array}$ & $\begin{array}{c}\text { Límite superior del } \\
\text { intervalo [seg] }\end{array}$ & Pendiente & Constante \\
\hline $18000(5: 00 \mathrm{~h})$ & $25200(7: 00 \mathrm{~h})$ & 0.5 & 80 \\
\hline $25200(7: 00 \mathrm{~h})$ & $32400(9: 00 \mathrm{~h})$ & 0.8 & 110 \\
\hline $32400(9: 00 \mathrm{~h})$ & $39600(11: 00 \mathrm{~h})$ & 1.1 & 150 \\
\hline $39600(11: 00 \mathrm{~h})$ & $46800(13: 00 \mathrm{~h})$ & 1.3 & \\
\hline
\end{tabular}

\subsection{Análisis y discusiones}

Partiendo del caso de estudio propuesto, el objetivo es entonces, encontrar la ruta de las recogidas (secuencia de enfriadoras a visitar), así como también el tiempo de inicio del tour, tal que se minimicen los costos, que como se menciona anteriormente, se componen de costos por espera, costos por tiempo de servicio y penalización por pérdida de ventas. Además, la función objetivo del modelo matemático plantea también el costo por consumo de gasolina en los desplazamientos entre nodos, de manera que se requiere considerar el parámetro de consumo de gasolina por tiempo de recorrido que es de $0.0011 \mathrm{lt} / \mathrm{seg}$ (AEOC, 2017). Adicionalmente es necesario considerar una velocidad promedio del vehículo para obtener el parámetro tij (tiempo de viaje del nodo i al j) a partir de la matriz de distancias que se obtiene de las coordenadas de los nodos, que se encuentran en Riascos \& Taimal (2019), y en este trabajo dicha velocidad promedio se asume de $45 \mathrm{Km} / \mathrm{h}$.

El modelo propuesto fue programado en $\mathrm{AMPL}^{\circledR}$ y el caso de estudio se resolvió usando el solver CPLEX, y la secuencia de enfriadoras a vistar están dadas por $\{0,26,20,11,5,8,23,1,17,15,7,2,10,19,6,14,18,12,16$, $24,25,21,13,4,3,22,9,0\}$, donde 0 es la galería. El costo óptimo de la ruta fue de $\$ 55093.5$ con un tiempo óptimo de inicio de ruta de $10488.22 \mathrm{seg}$, la duración del recorrido de 11111.77 seg y distancia total recorrida de $138.89 \mathrm{~km}$.

Figura 5

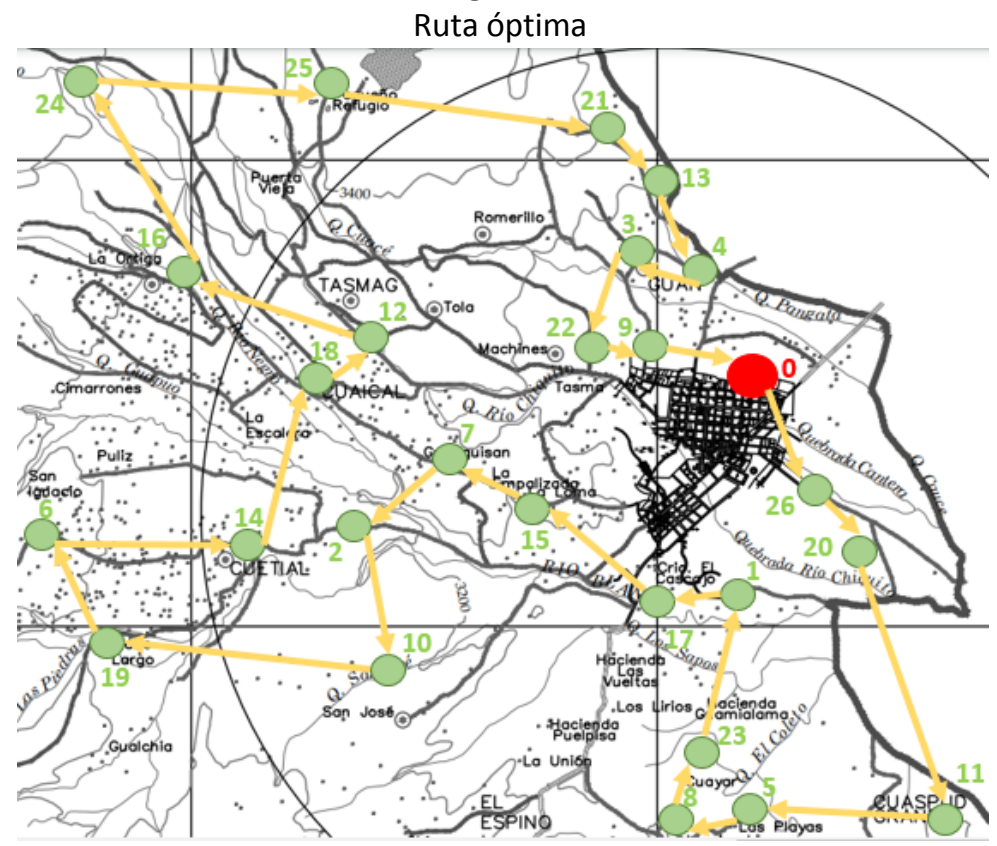




\section{Análisis de escenarios}

Se realiza un análisis de sensibilidad en el marco de tres escenarios que se explicrán seguidamente, y que desde el contexto practico podrían estar asociados con: cambios tecnológicos en los vehículos, variaciones en la disponibilidad de mano de obra para descargue y cambios en las percepciones de los clientes frente a bajos niveles de servicio. Las variaciones se plantean en factores de $0 \%$ a $300 \%$ avanzando en $20 \%$, es decir, factores de $0 \%, 20 \%, 40 \%, \ldots, 300 \%$, respecto al valor del parámetro inicialmente propuesto en el caso de estudio.

Cambios tecnológicos en los vehículos: el parámetro considerado en este primer escenario del análisis de sensibilidad es el consumo de gasolina de la unidad de refrigerado (cguf), cuya variación tiene un sentido práctico en términos de los cambios tecnológicos de los vehículos refrigerados que generen menores consumos. En la Figura 6 se muestra el comportamiento de la función objetivo (curva amarilla), así como cada uno de las componentes de esta. Para los cambios en el parámetro cguf, el impacto sobre los costos por consumo en el recorrido es nulo (curva azul), es decir, no hay ningún impacto en la ruta de las recogidas.

También en la Figura 6 se observa que los elementos de costo que se impactan son el costo por consumo de la unidad de frío (curva naranja) y los costos por pérdida de ventas (curva gris). Resulta interesante observar que cuando el factor de variación del parámetro se encuentra entre 0 y $60 \%$, el costo por pérdida de ventas es nulo, mientras que el costo por consumo de la unidad de frio es creciente, pero a partir del factor del $80 \%$ en adelante, el modelo trata de compensar ambos elementos de costo, lo cual se realiza a través de encontrar el mejor tiempo de inicio del tour, como se observa en la Figura 7, que muestra como del factor del $80 \%$ en adelante el tiempo de inicio es mayor que el de los factores anteriores. Aquí se observa la importancia del tiempo de inicio optimo.

Figura 6.

Costos escenario 1

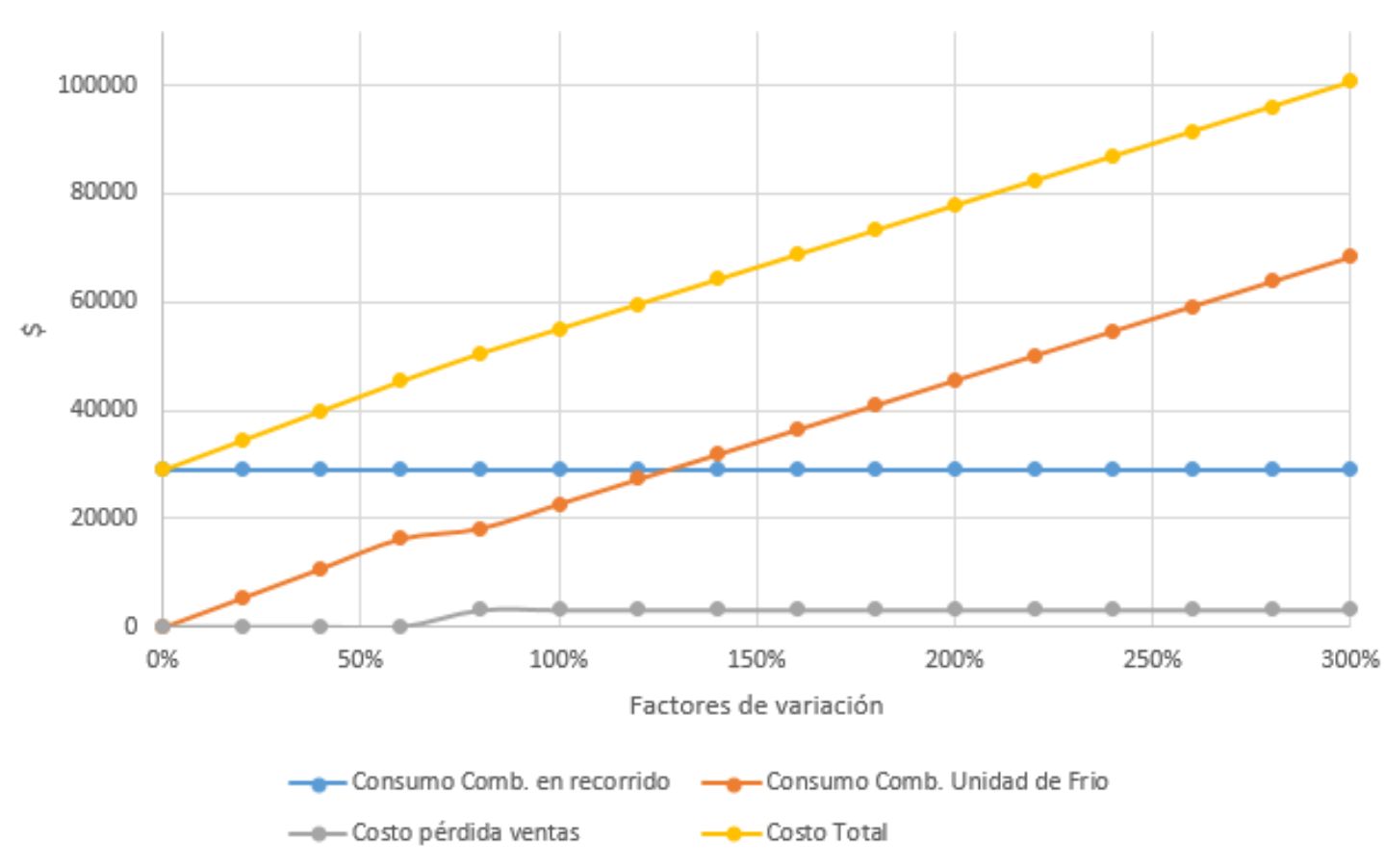


Figura 7

Tiempo de inicio escenario 1

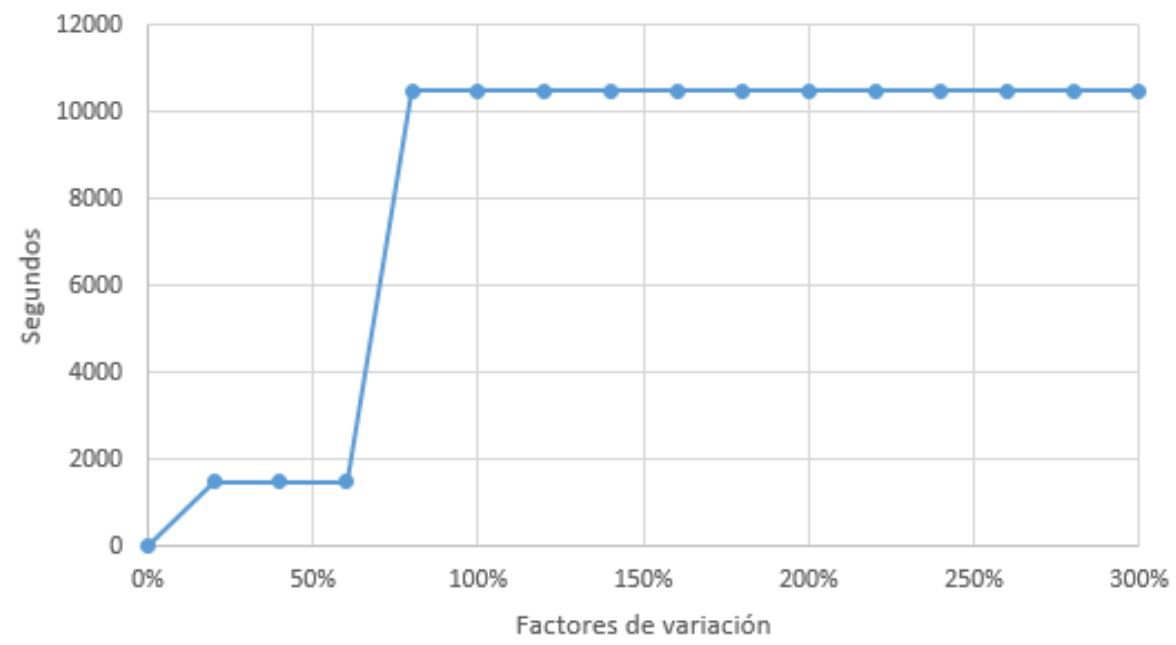

Variaciones en la disponibilidad de mano de obra para descargue: el parámetro que se varía en el segundo escenario es el tiempo de servicio tiempo dependiente (tstd) para cada uno de los intervalos de tiempos de servicio (conjunto k). En la práctica es común que los tiempos de servicio varíen según la disponibilidad de mano de obra para el descargue de los vehículos. Las variaciones se hacen multiplicando cada factor para los valores de cada intervalo $k$, obteniéndose los resultados de la Figura 8 que muestra el efecto de las variaciones del parámetro en los costos.

Similarmente al escenario anterior, en este caso tampoco ocurren cambios en la ruta de las recogidas y por consiguiente el costo de consumo de combustible en el recorrido permanece constante (curva azul). También en este escenario se observa el fenómeno de compensación entre el costo por consumo de la unidad de frio (curva naranja) con el costo por pérdida de ventas (curva gris), específicamente cuando el factor de variación es del $140 \%$, que es cuando la Figura 9 también registra una variación en el tiempo de inicio, reforzando el hecho de que la compensación entre esos dos costos se da ajustando el tiempo de inicio del tour. También cabe mencionar que en este segundo escenario el costo por pérdida de ventas tiene mayor sensibilidad al parámetro, lo cual se sustenta en la tendencia creciente de la curva gris y se explica por el hecho de que el costo por pérdida de ventas está en función del tiempo de finalización del descargue, y por consiguiente, la duración del descargue lo impacta.

Para este escenario se observa que para los factores de variación de 0 y $20 \%$ en el parámetro tstd se mantienen en cero los costos por pérdida de ventas asumiendo solo costos por recorrido y por refrigeración. Sin embargo, a partir de los factores de variación de $40 \%$, es decir, a mayores tiempos de servicio, se trata de compensar el impacto de dichos tiempos en los costos de refrigeración, con los costos de pérdida de ventas, de manera que el modelo prefiere ubicarse en el intervalo con menor tiempo de servicio así ello implique incurrir en pérdida de ventas, análogamente al escenario anterior. Adicionalmente se observa un comportamiento interesante cuando el factor de variación es del $140 \%$ porque allí el crecimiento del costo por pérdida de ventas, trata de equilibrarse a través de un menor tiempo de inicio, pero garantizando que se caiga en el segundo intervalo de menor costos, que es el primero en la Figura 2, lo cual requiere un tiempo de inicio bajo, tal como muestra la figura 9. 
Figura 8

Costos escenario 2

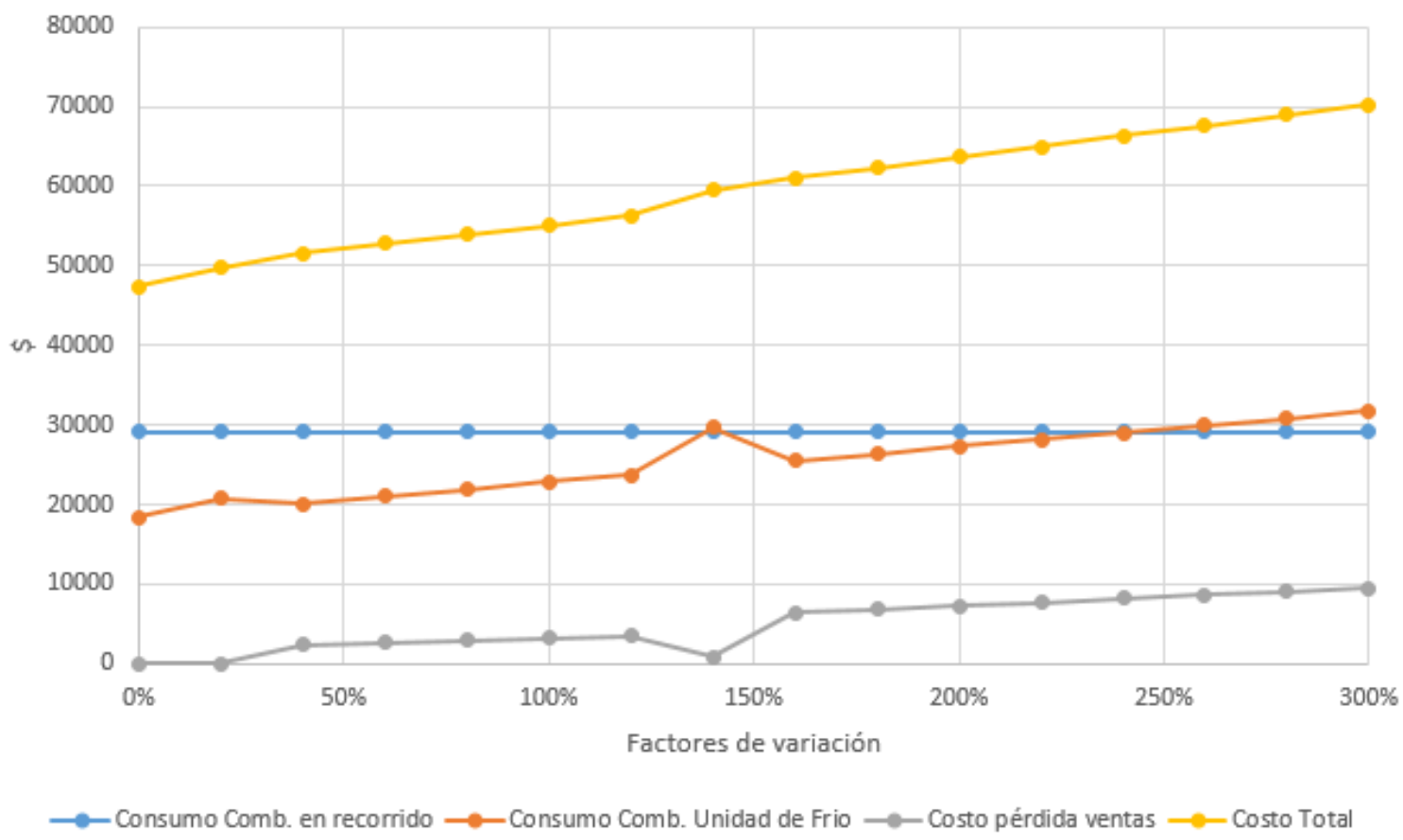

Figura 9

Tiempo de inicio escenario 2

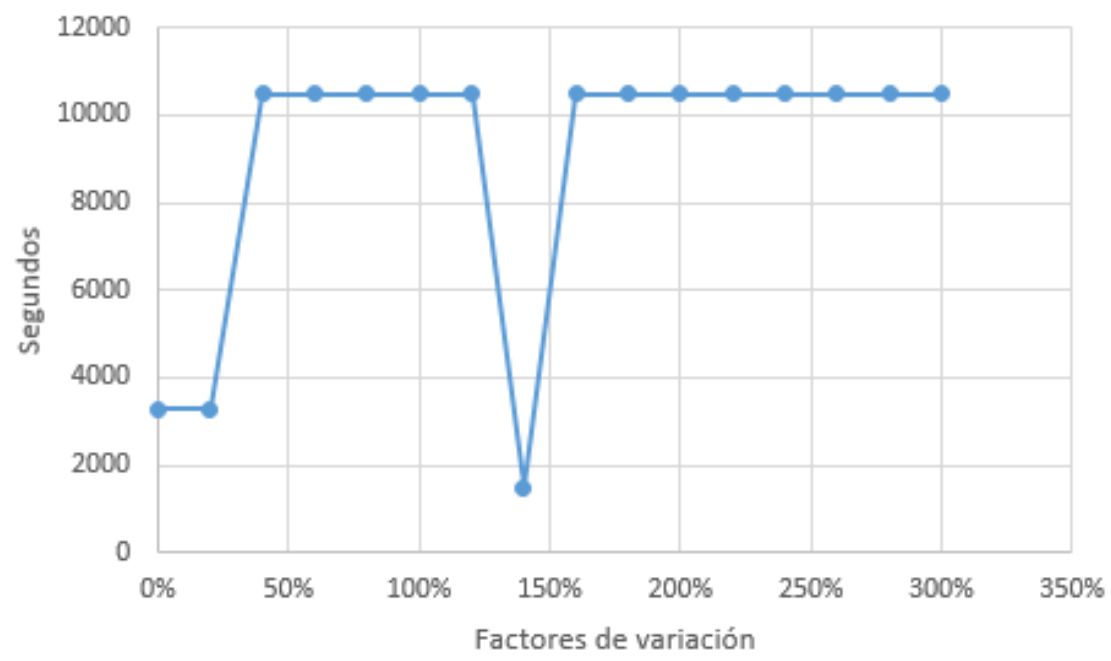

Cambios en las percepciones de los cliente frente a bajos niveles de servicio: finalmente el tercer escenario propone variar el costo por pérdida de ventas, que como se puede observar en la función objetivo del modelo, se compone de un costo fijo y de un costo variable en el que se consideran las pendientes de las rectas, así que para efectos del análisis de sensibilidad se considera primero la variación del elemento de costo fijo ( $p p v$ ) para cada uno de los intervalos, generando la curva gris de la Figura 12 que se denota como escenario 3a, y luego se realiza la variación de la pendiente (pend) para cada intervalo de lo cual se obtiene la curva amarilla de la Figura 12, escenario 3b.Resulta pues evidente que para el tercer escenario, la función objetivo es poco sensible a las variaciones de los elemento del costo por pérdida de ventas, sin embargo, el elemento de la pendiente (pend) muestra un mayor impacto que el elemento de costo fijo ( $p p v)$. 
Es importante observar que en las variaciones del elemento de pendiente se vuelve a observar la compensación entre el costo por consumo de la unidad de frio y el costo por pérdida de ventas, a través del tiempo de inicio del tour (T0), tal como se observa en la Figura 10 y la Figura 11. En esta compensación se vuelve a observar la lógica de que los tiempos de inicio altos conllevan a llegadas tardías del vehículo al deposito, por lo que se generan pérdida de ventas, pero esos costos por pérdida de ventas se compensan con costos más bajos en la refrigeración, porque el intervalo de menor tiempo de servicio es precisamente el más tardío. Sin embargo, en este escenario se observa que cuando los costos por pérdida de ventas aumentan considerablemente (factores de variación mayores al 140\%), el modelo decide no incurrir en más perdidas de ventas así eso le implique mayores costos por refrigeración durante el tiempo de servicio, y por lo tanto disminuye el tiempo de inicio del tour.

Figura 10

Costo escenario 3

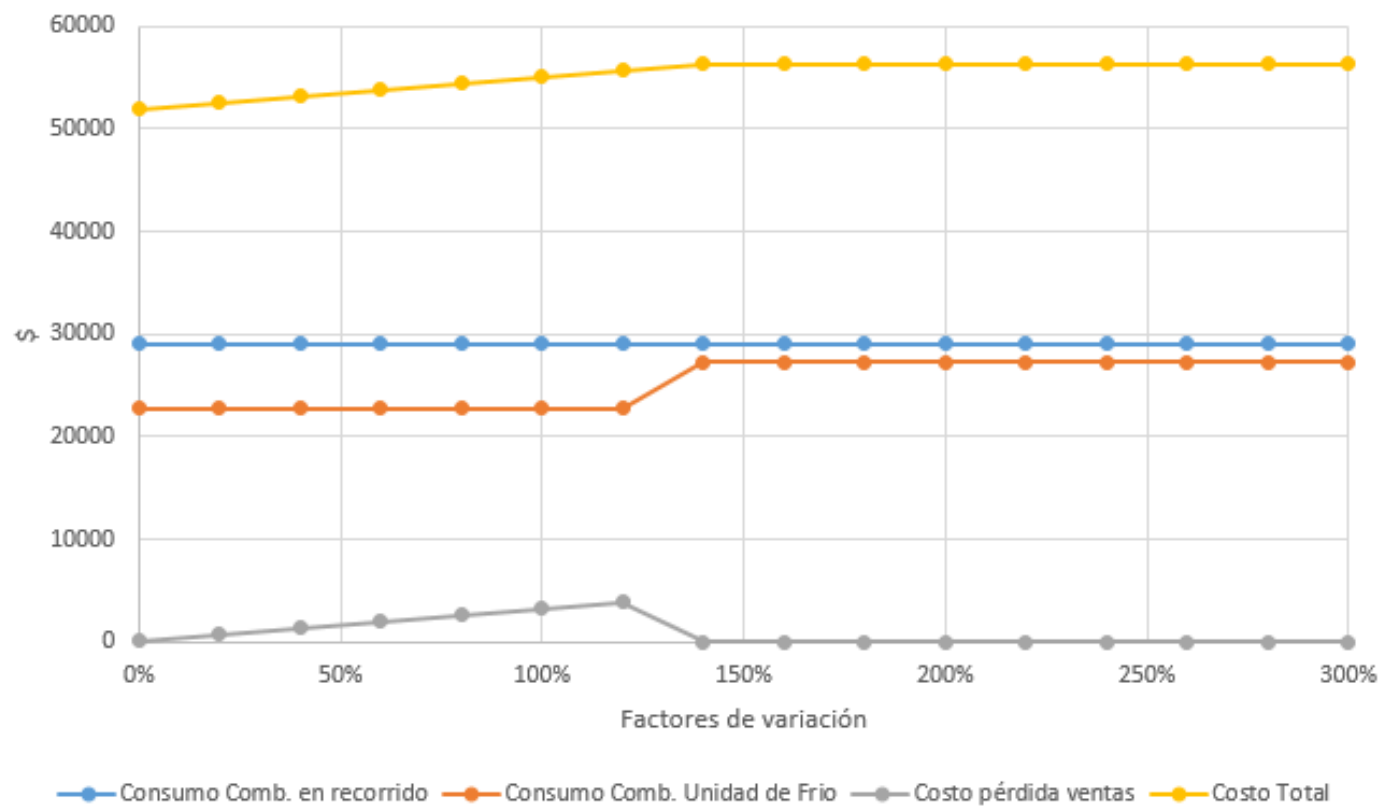

Figura 11

Tiempo de inicio escenario 3

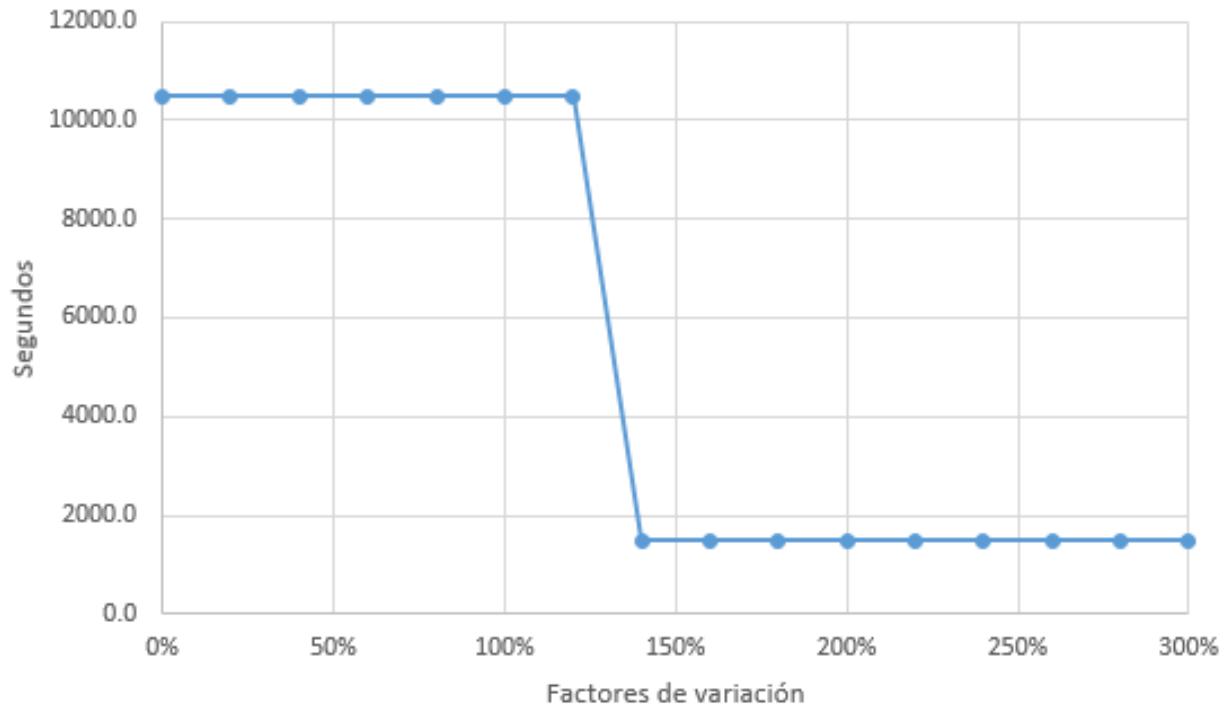


En la Figura 12 se resumen los comportamientos de la función objetivo para cada uno de los escenarios propuestos. En ella se muestra que la intersección de las curvas se da cuando el factor de variación es el 100\%, es decir, cuando los parámetros quedan con los valores originales del caso, y por tanto las soluciones de los escenarios coinciden con la solución óptima del caso obtenida anteriormente. Se recuerda que las variaciones ocurren cuando se disminuyen los valores de los parámetros (factores menores al 100\%) o se aumentan (factores superiores al 100\%).

Resulta por lo anterior relevante observar el impacto o la sensibilidad en funcion objetivo de las perturbaciones de los parámetros, al afectarlos con factores de variación. Se puede observar que el modelo es poco sensible a los costos por pérdida ventas ya que las curvas de los parámetros asociados a dichos costos (amarilla y gris en la Figura 12) son las que tienen las menores tasas de variación. Similarmente se observa que las tasas de crecimiento y decrecimiento de la curva azul son mayores a los del resto de curvas, indicando que la función objetivo del caso de estudio tiene mayor sensibilidad a los consumos de la unidad de refrigerado del vehículo, lo cual es razonable, puesto que el consumo de gasolina de la unidad de frio es un parámetro que impacta tanto los recorridos del tour, como las esperas y los tiempos de descargue.

Figura 12

Análisis de sensibilidad

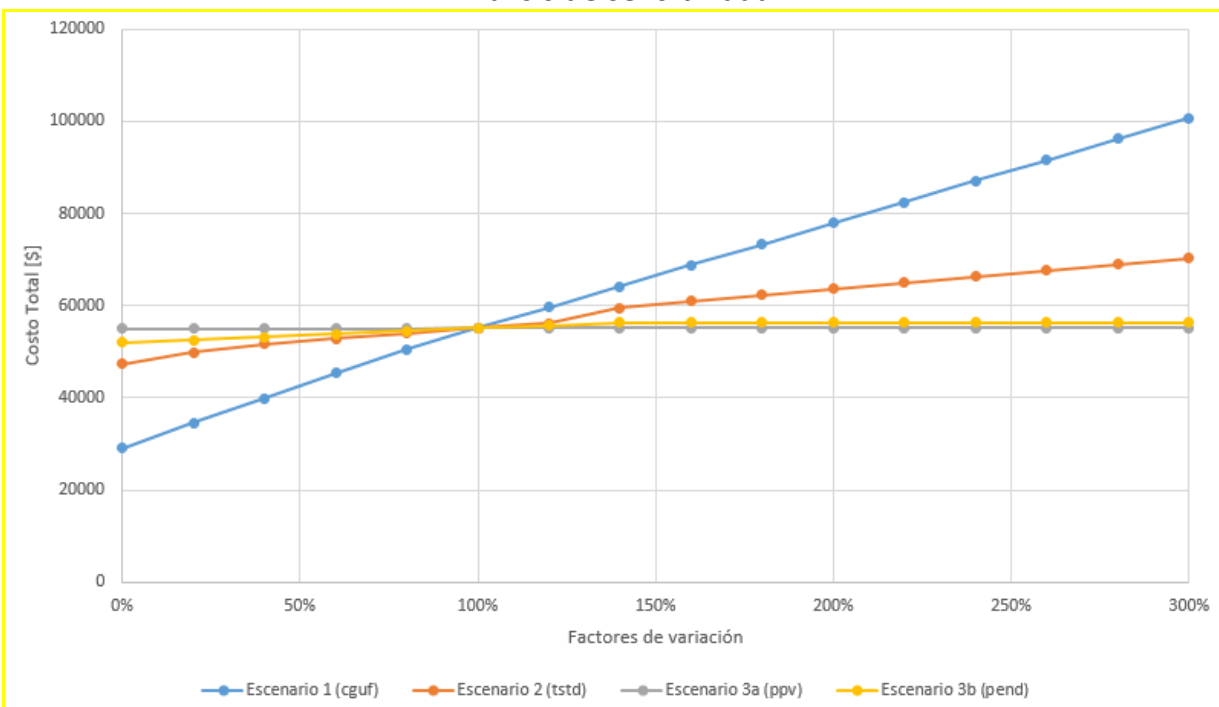

\section{Análisis sobre el tiempo de inicio}

En el análisis de sensibilidad de los escenarios anteriores se pudo observar el papel importante del tiempo de inicio (TO) en la estructura de costos de la función objetivo, y para profundizar más al respecto, a continuación se propone cambiar la naturaleza del To en el modelo, que originalmente es una variable de decisión, parametrizándolo para observar los impactos que tiene en la función objetivo ante su cambio, como se muestra en la Figura 13.

Se puede observar como la función objetivo (curva azul claro) va tomando distintos valores en relación a distintos valores del tiempo de inicio T0, mostrando una sensibilidad considerable e incluso dibujando una curva con óptimos relativos. Dichas variaciones de la función objetivo es el reflejo de las variaciones de sus componentes de costos: costo por consumo de combustible en recorrido (curva gris), costo por consumo de combustible de la unidad de frio (curva amarilla) y costo por pérdida de ventas (curva azul oscuro). Frente al comportamiento de esas componentes en relación al tiempo de inicio T0 se puede observar que la componente que en mayor medida se impacta por el tiempo de inicio es la del costo por pérdida de ventas, mientras que la más estable de las componentes es la del costo por consumo en el recorrido, ya que en la mayoría de los casos la ruta no se cambia. 
Sin embargo, es muy importante observar que en esa curva gris existe un intervalo entre los valores de T0 de 7980 y 10430 segundos, en el que la ruta cambia generando un pico en dicho intervalo, es decir, que en ese intervalo es mejor una ruta con mayor duración para lograr una llegada más tardía que compense los componentes de costos de la función objetivo. En este caso con el T0 fijo o parametrizado, la llegada más tardía no se puede lograr a través del tiempo de inicio del tour, ya que este es un parámetro, y por tanto el modelo busca hacerlo a través de la duración del tour, así ello le genere mayores costos de recorrido, porque al compensarse con los otros costos se obtenga la minimización del costo total.

Lo anterior muestra la incidencia relevante del momento de inicio de la ruta (TO) en los costos de este tipo de problemas, y por tanto, resulta importante que en la práctica la programación de operaciones tenga en cuenta ese elemento temporal para no caer en ineficiencias no deseadas como se muestra en la Figura 13.

Figura 13

Impacto del momento de inicio en los costos

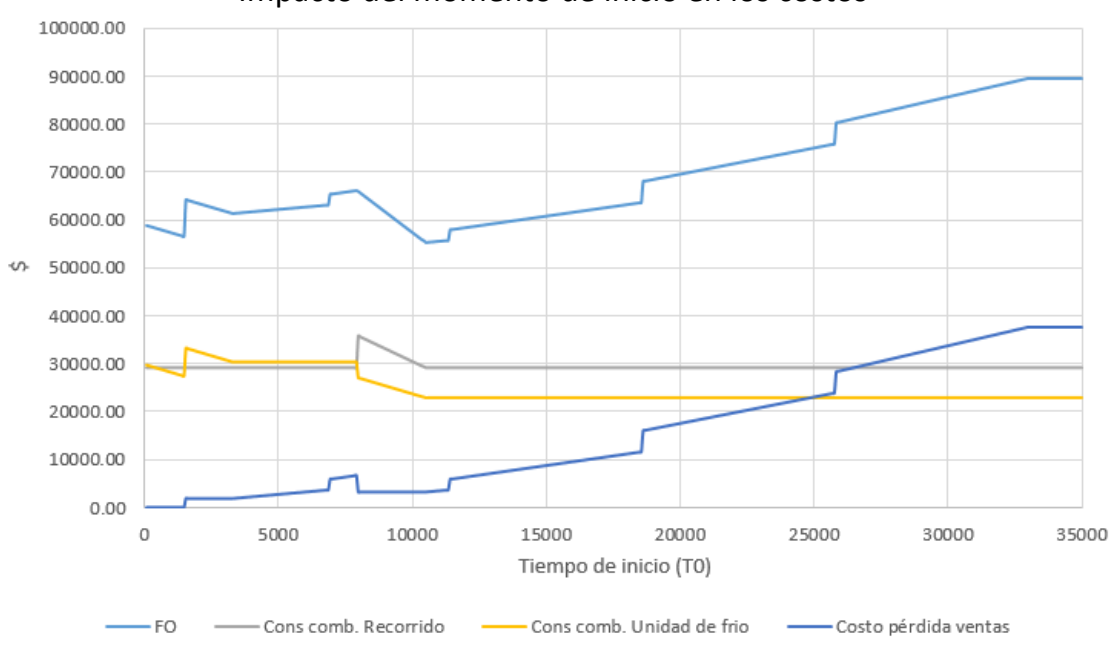

\section{Conclusiones}

El modelo propuesto involucra aspectos relevantes para los problemas de recogidas de productos refrigerados que no se han tratado de manera conjunta en los artículos revisados en la literatura. Dichos aspectos implican que el alcance de las decisiones no se limita únicamente a la secuencia de visitas (definición del tour) sino que también es necesario definir el tiempo de inicio del tour. Respecto a la importancia de dicho tiempo de inicio se logra mostrar el considerable impacto de éste frente al costo total, siendo que para una misma secuencia de visitas, el tiempo de inicio de dichas visitas marca una relevante importancia en el costo de la operación, lo cual amerita su consideración dentro de los procesos de optimización, tal y como se hace en el modelo propuesto. Se pudo validar que los tiempos de servicio o descarga tiempo-dependientes son una aproximación realista y sugerida de considerar en entornos practicos. Se logra probar el modelo propuesto en un problema de relevancia en el contexto colombiano como es el de la cadena de la leche. Se resuelve el problema a través de la implementación en $A M P L \circledR$ y la optimización con el solver CPLEX, sin embargo podrían explorarse estrategias de solución diferentes en potenciales instancias de mayor tamaño.

\section{Referencias bibliográficas}

AEOC. (2017). Guía de cálculo de la huella de carbono del transporte de mercancías por carretera, 76. Retrieved from http://www.comunidadism.es/wp-content/uploads/downloads/2019/03/Guía-Técnica-HCmercancías.pdf

Ballou, R. (2004). Logística. Administración de la cadena de suministro (Quinta Edi). Pearson Educación. 
Cacchiani, V., Contreras-Bolton, C., \& Toth, P. (2020). Models and algorithms for the Traveling Salesman Problem with Time-dependent Service times. European Journal of Operational Research, 283(3), 825-843. https://doi.org/10.1016/j.ejor.2019.11.046

FAO. (2021). Producción y productos lácteos: recogida y transporte. Retrieved from http://www.fao.org/dairyproduction-products/processing/collection-and-transport/es/

Giesen, R., Mahmassani, H. S., \& Jaillet, P. (2005). Strategies for online inventory routing problem under realtime information. Transportation Research Record, (1923), 164-179. https://doi.org/10.3141/1923-18

Giesen, R., Mahmassani, H. S., \& Jaillet, P. (2010). Logistics in real-time: Inventory-routing operations under stochastic demand. Lecture Notes in Economics and Mathematical Systems (Vol. 619). https://doi.org/10.1007/978-3-540-92944-4_7

Gutin, G., \& Punnen, A. (2003). The traveling salesman problem and its variations. Springer. https://doi.org/10.16309/j.cnki.issn.1007-1776.2003.03.004

Hashimoto, H., Yagiura, M., \& Ibaraki, T. (2008). An iterated local search algorithm for the time-dependent vehicle routing problem with time windows. Discrete Optimization, 5(2), 434-456. https://doi.org/10.1016/j.disopt.2007.05.004

Laporte, G. (2010). A concise guide to the Traveling Salesman Problem. Journal of the Operational Research Society, 61(1), 35-40. https://doi.org/10.1057/jors.2009.76

Malandraki, C., \& Daskin, M. (1992). Time dependent vehicle routing problems: formulations, properties and heuristic algorithms. Transportation Science, 26(3), 185-200.

Miller, C. E., Zemlin, R. A., \& Tucker, A. W. (1960). Integer Programming Formulation of Traveling Salesman Problems. Journal of the ACM (JACM), 7(4), 326-329. https://doi.org/10.1145/321043.321046

Moosavi, A., \& Nikfarjam, A. (2019). A multi-path routing-inventory problem for a closed-loop supply chain considering the heterogeneous fleet of vehicles. International Journal of Sustainable Engineering, 12(3), 174-188. https://doi.org/10.1080/19397038.2019.1566412

Mukhopadhyay, A., \& Goswami, A. (2016). Stockout aversion in retailing supply chain using newsvendor models. International Journal of Mathematics in Operational Research, 8(2), 185-202. https://doi.org/10.1504/IJMOR.2016.074854

Orejuela, J. P., \& Hernández, G. (2019). Time Dependent School Bus Routing. Revista Espacios, 40(15), 24.

Riascos, Z., \& Taimal, O. (2019). Diseño de un sistema de recolección y almacenamiento de leche para el municipio de Cumbal. Universidad del Valle.

Taş, D., Gendreau, M., Jabali, O., \& Laporte, G. (2016). The traveling salesman problem with time-dependent service times. European Journal of Operational Research, 248(2), 372-383.

https://doi.org/10.1016/j.ejor.2015.07.048

Esta obra está bajo una Licencia Creative Commons Attribución-NoCommercial 4.0 International

$(\mathrm{Cc}) \mathbf{B Y}$ B-NO 